\title{
Nursing Experience of CT Examination in a Case of Critical Elderly Male Patients with Neck Injury: A Case Report
}

\author{
Sihua Liang, Xueke Wen, Yanan Chi, Yongguang Chen, Lin Qiu, Chunliu Luo* \\ Radiology Department, The First Affiliated Hospital, Jinan University, Guangzhou, China \\ Email address: \\ 13533123111@139.com (Sihua Liang),407598271@qq.com (Xueke Wen),251050600@qq.com (Yanan Chi), \\ 708857674@qq.com (Yongguang Chen),jnuql@qq.com (Lin Qiu),jnuchll@163.com (Chunliu Luo) \\ *Corresponding author
}

\section{To cite this article:}

Sihua Liang, Xueke Wen, Yanan Chi, Yongguang Chen, Lin Qiu, Chunliu Luo. Nursing Experience of CT Examination in a Case of Critical Elderly Male Patients with Neck Injury: A Case Report. American Journal of Nursing Science. Vol. 9, No. 4, 2020, pp. $248-251$.

doi: 10.11648/j.ajns.20200904.25

Received: June 16, 2020; Accepted: July 1, 2020; Published: July 13, 2020

\begin{abstract}
We present a 63-years-old male who are accidentally fell down while riding a bicycle and was inserted into his neck by a $4 \mathrm{~cm}$ thick bamboo pole on the morning of August 30, 2019. In rescue process, patient's mental status is bad, that the patient's state of mind is in tension, fear, anxiety and restlessness. the patient takes out stitches on September 9, 10 days rehabilitation hospital discharge. As the patient had pale complexion, slightly shortness of breath, temporarily stable vital signs, and multiple blood scabs formed throughout the body, he underwent neck CT plain scan, enhancement, 3D reconstruction, chest CT plain scan, enhancement and 3D reconstruction. After that, the patient was sent to operating room, he received suitable treatment. Furthermore, the sharing of patient information was a great help in this rescue. The local hospital provides enough patient information to our CT examine. the difficulty of this rescue lies in whether the patient can complete CT examination smoothly. In nursing aspect, we not only fixed the patient's body position and keep the patient's breathing open to prevent secondary injury but also provide psychological counseling and comfort to patients so that we ensure that the mental health of the patient is good.
\end{abstract}

Keyword: CT, Nursing, Neck Injury

\section{Introduction}

Trauma is one of leading cause of death worldwide, that neck injury is an important part in trauma cases, with a higher incidence in low- and middle-income countries than in high-income countries $[1,2]$. Base on neck injury report of Saharan Africa, more deaths in this place compare with anywhere else in the world [3]. Although neck injury in patients who have suffered blunt or penetrating trauma are not frequent, it had high morbidity and mortality rates in trauma patients $[4,5]$. Neck injury is clinically divided into closed trauma and open trauma. Neck injury is one of the emergency diseases in otorhinolaryngology head and neck surgery. The symptoms of neck injury contain massive hemorrhage, Shock and dyspnea $[6,7]$.

Over the past several decades, many technological advances in the realm of workflow, they create an increasingly complex work environment for radiologists and radiology technologists $[8,9]$. The CT image plays a critical role in the diagnosis and management of a wide range of diseases. In addition, complexity of CT services tailored to the individual needs of each patient continues to grow [10-13]. We present the case of a 63-year-old man who was stabbed in the neck by $4 \mathrm{~cm}$ thick bamboo pole, the old man suffers pain, bleeding, and limited mobility. We discuss nursing cooperation in CT scanning process of this patient.

\section{Case Report}

A 63-years-old male who are accidentally fell down while riding a bicycle and was inserted into his neck by a $4 \mathrm{~cm}$ thick bamboo pole on the morning of August 30, 2019 (Figure 2). People call the fireman to save him, that they saw off bamboo poles and sent to local hospitals. However, the patient's injuries were too severe, the medical staff of local hospital cannot deal with this injuria, so they only make simple bandage hemostasis to patient (Figure 1). After that, the patient is sent to The First Affiliated Hospital of Jinan 
University. As the patient had pale complexion, slightly shortness of breath, temporarily stable vital signs, and multiple blood scabs formed throughout the body, he underwent neck CT plain scan, enhancement, 3D reconstruction, chest $\mathrm{CT}$ plain scan, enhancement and $3 \mathrm{D}$ reconstruction. After that, the patient was sent to operating room, he received general anesthesia downlink neck open wound exploration, neck vascular probe, neck vascular suture, neck wound debridement suture, postoperative intensive care, transferred to the ICU for anti-inflammation and stop bleeding, and rehydration symptomatic treatment. Finally, the patient takes out stitches on September 9, 10 days rehabilitation hospital discharge.

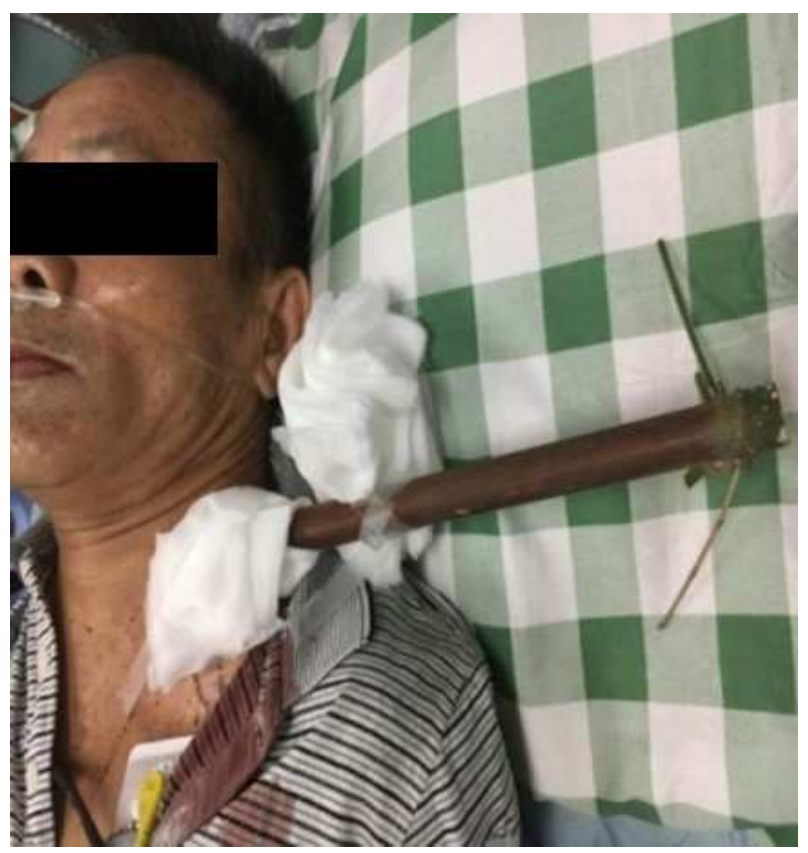

Figure 1. body position before checking.

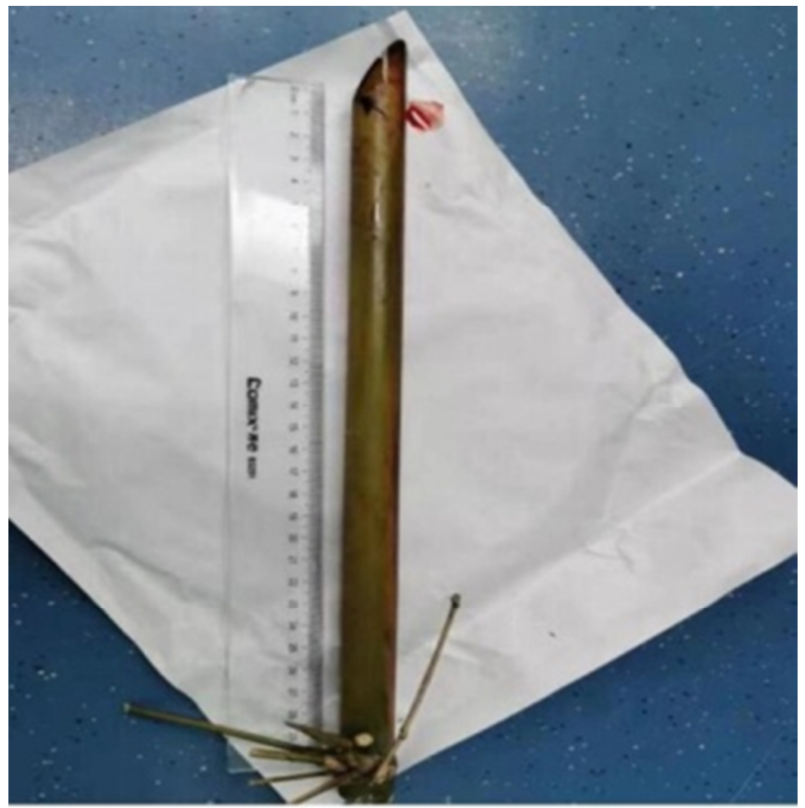

Figure 2. Bamboo foreign body $4 \times 40 \mathrm{~cm}$.

\subsection{Equipment and Nursing Coordination}

The equipment we use includes the following: Plain NECK CT scan (320-row 640-slice dynamic volume), 3D reconstruction and plain chest CT scan were performed. 20G indwelling needle venipuncture, iodine contrast agent and single cylinder high-pressure injection $(3.0 \mathrm{~mL} / \mathrm{s}$, total $70 \mathrm{ml})$. In nursing coordination, inspect the process to provide complete, professional and meticulous care.

\subsection{Examination and Nursing}

\subsubsection{Preparation Before Inspection}

We have prepared a special channel for this patient, so he receives faster rescue. In addition, we prepare all rescue drugs and articles, and arrange the examination as soon as possible.

\subsubsection{Mental Nursing}

In rescue process, patient's mental status is bad, that the patient's state of mind is in tension, fear, anxiety and restlessness. The nurse soothed and explained for the patient, they can avoid the patient because of emotional agitation and restlessness will lead to bamboo pole displacement, fall off, leading to massive bleeding.

\subsubsection{Keep the Patient's Airway Clear}

We prepare the suction device to keep the patient's airway clear, because ventilatory disturbance is a faster cause of death than hemorrhagic shock, mainly caused by head, neck, maxillofacial and thoracic trauma [10].

\subsubsection{Care Before Examination}

Pre-examination care includes the following: (1) Check the work before the inspection. The receiving nurse carefully checks the patient's name, gender, age, examination items and locations, carefully checks the patient's diagnosis and medical history, and learns that the patient's physical condition is consistent with the examination. (2) Patient evaluation. The CT equipment has a narrow space and the scanning round hole is limited. The technician and nurse should evaluate the patient's condition to ensure that he can pass through the scanning hole smoothly to ensure enough scope space to prevent the bamboo pole from shifting and falling off and avoid secondary injuries. (3) Equipment to measure. Since the examination bed moved back and forth during scanning to ensure that the bamboo pole was not disturbed, the nurse immediately used a soft ruler to measure the bamboo pole and the scanning hole in the neck, and made correct adjustments to ensure adequate scanning range space. (4) Keep your respiratory tract clear. The patient was given flow oxygen to relieve discomfort. (5) Fix the patient. The patient had a $4 \mathrm{~cm} \times 40 \mathrm{~cm}$ bamboo foreign body inserted in the neck, which was in an active state. The patient's neck was fixed to avoid the bamboo pole swinging and neck blood vessels being damaged (6) Establish a good nurse-patient relationship. Strengthen the emotional communication and communication with patients and their families to gain the trust of patients. (7) Look for causes of anxiety. (8) Remove metal objects. Quickly assess whether the patient has metal objects in the neck and chest 
area and remove them as soon as possible. Do not undress if there are metal fasteners and zippers to avoid secondary damage. (9) Ask about your medical history and allergies. The patient and his family members were asked whether the patient had a history of food and drug allergy, a history of hyperthyroidism, and normal renal function. Contraindications were excluded and informed consent was signed. (10) Indwelling peripheral vein needle.

\subsubsection{Nursing During Examination}

Nursing during examination includes the following: (1) Place the patient in the right position for examination. (2) Cooperate to inform during inspection. Patients were told to keep quiet during examination to avoid movement artifacts, which would affect the imaging diagnosis. (3) Start the high-pressure syringe. Connect the high-pressure syringe to keep the flow open and secure. (4) Watch closely. Nurses are not allowed to leave during the high-pressure injection and should closely observe adverse drug reactions and discomfort.

\subsubsection{Care After Examination}

Care after examination contains the following: (1) Inquiry and observation. Ask the patient if there is any discomfort, check whether there is drug extravasation, quickly separate the high-pressure syringe, and explain matters needing attention to the patient and his/her family. (2) Notice matters needing attention. Due to the critical condition, and told the doctor in charge to observe closely within 15 minutes to avoid the risk of delayed iodine contrast allergy.

\section{Discussion}

The result of examine indicated that the foreign body was inserted in the left neck, with blood and gas accumulation in the neck. The foreign body was located between the common carotid artery and the jugular vein. The distance between the foreign body's tip and the common carotid artery was about $1.0 \mathrm{~cm}$ (Figure 3). In rescue process, assessment and preparation before inspection, cooperation during inspection and observation after inspection are important in CT examination procedure. In addition, professional skills and specialized nursing knowledge are also very important. In particular, the evaluation and fixation before CT examination ensures that the professional measuring bamboo pole can pass through the scanning hole smoothly and ensure adequate scanning range space.

Furthermore, the sharing of patient information was a great help in this rescue. The local hospital provides enough patient information to our CT examine. Nowadays, most medical institutions have established patient information sharing systems or platforms, it provided a lot of support to treatment, especially rescue $[14,15]$.

In summary, the difficulty of this rescue lies in whether the patient can complete CT examination smoothly. In nursing aspect, we not only fixed the patient's body position and keep the patient's breathing open to prevent secondary injury but also provide psychological counseling and comfort to patients so that we ensure that the mental health of the patient is good.

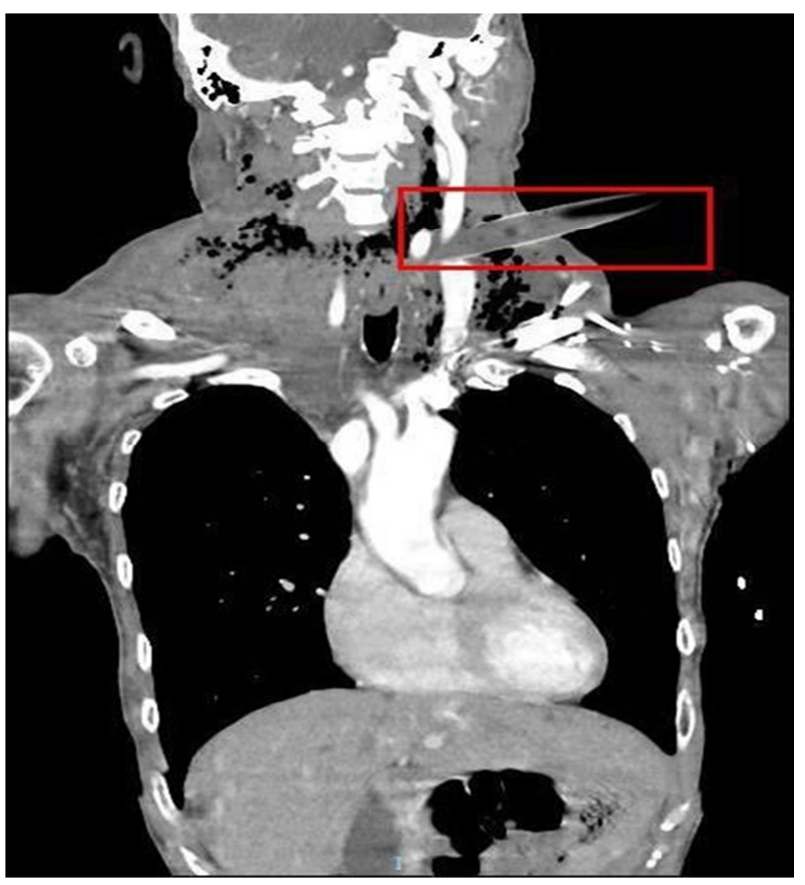

Figure 3. Video images.

\section{References}

[1] Peden M, McGee K, Krug E. Injury-a leading cause of the global burden of disease 2000. Geneva: World Health Organization. 2016.

[2] Jubert P, Lonjon G, Garreau de Loubresse C. Complications of upper cervical spine trauma inelderly subjects. A systematic review of the liter-ature. Orthop Traumatol Surg Res. 2017: 301-312.

[3] Bowley DM, Khavandi A, Boffard KD, et al. The malignant epidemic-changing patterns of trauma. S Afr Med J. 2015; 92: 798-802.

[4] Nowicki JL, Stew B, Ooi E. Penetrating neck injuries: a guide to evaluation and management. Ann R Coll Surg Engl. 2018; 100: 6-11.

[5] Sethi RK, Kozin ED, Fagenholz PJ, Lee DJ, Shrime MG, Gray ST. Epidemiological survey of head and neck injuries and trauma in the United States. Otolaryngol Head Neck Surg. 2016; 151 (5): 776-84.

[6] Zhou XQ, Jia H, Lu J, et al. Nursing experience of an open laryngeal trauma patient. World latest medical information digest. 2016, 16 (91): 246-246.

[7] Ahmed A. Selective observational management of penetrating neck injury in Northern Nigeria. S Afr J Surg. 2019; 47 (3): $80-85$.

[8] Kansagra AP, Liu K, Yu JP. Disruption of radiologist workflow. Curr Probl Diagn Radiol 2016; 45 (2): 101-6.

[9] Boland GW. Enhancing CT productivity: strategies for increasing capacity. AJR AmJ Roentgenol. 2018; 191 (1): 314.

[10] Gao JM. Several important problems of trauma first aid [J]. Journal of trauma surgery. 2013; 15 (1): 1-4. 
[11] De Visschere P, Oosterlinck W, De Meerleer G, Villeirs G. Clinical and imaging tools in the early diagnosis of prostate cancer, a review. JBR-BTR. 2016; 93: 62-70.

[12] Richter M, Lintz F, Zech S, Meissner SA. Combination of Ped CAT weightbearing CT with pedography assessment of the relationship between anatomy-based foot center and force/pressure-based center of gravity. Foot Ankle Int. 2018; 39: $361-368$.

[13] Barg A, Bailey T, Richter M, de Cesar Netto C, Lintz F, Burssens A, et al. Weightbearing computed tomography of the foot and ankle: emerging technology topical review. Foot Ankle Int. 2018; 39: 376-386.

[14] Ferraris VA, Bolanos M, Martin JT, Mahan A, Saha SP. Identification of patients with postoperative complications who are at risk for failure to rescue. JAMA Surg. 2016; 149: 1103-1108.

[15] Massarweh NN, Anaya DA, Kougias P, Awad SS, Berger DH. Variation and impact of multiple complications on failure to rescue after inpatient surgery. Ann Surg. 2017; 266: 59-65. 